\title{
Ecouter et dire en médecine, une approche psycho-sociale : utiliser la simulation pour former les futurs médecins à la relation thérapeutique
}

Ralph Balez, Antonia Blanié et Alexandre Eghiaian

\section{QpenEdition}

\section{Journals}

Édition électronique

URL : http://journals.openedition.org/trema/3642

DOI : $10.4000 /$ trema.3642

ISSN : 2107-0997

Éditeur

Faculté d'Éducation de l'université de Montpellier

Édition imprimée

Date de publication : 1 décembre 2017

Pagination : 81-101

ISBN : 979-10-96627-03-5

ISSN : 1167-315X

\section{Référence électronique}

Ralph Balez, Antonia Blanié et Alexandre Eghiaian, «Ecouter et dire en médecine, une approche psycho-sociale : utiliser la simulation pour former les futurs médecins à la relation thérapeutique », Tréma [En ligne], 47 | 2017, mis en ligne le 01 février 2018, consulté le 30 avril 2019. URL : http:// journals.openedition.org/trema/3642 ; DOI : 10.4000/trema.3642

Ce document a été généré automatiquement le 30 avril 2019

Trema 


\title{
Ecouter et dire en médecine, une approche psycho-sociale : utiliser la simulation pour former les futurs médecins à la relation thérapeutique
}

\author{
Ralph Balez
}

Remerciements :

Les médecins qui ont participé à réaliser les séances de simulation et qui ont apporté leurs multiples connaissances et savoirs faire au dispositif: Vincent Allano, Patrick Balouet, Christian Berthou, Jean-Michel Boles, Amélie Calvez, Sophie Cusset, Florence Dalbies, Jean-Dominique Dewitte, Quentin Durand-Morreau, Anne-Sophie Gut, Lionel Touffet, Erwan L'Her, Jean-Yves Le Reste, Brice Loddé, Anne Renault, Romuald Seizeur, François Simon, Jacques Sizun, Michel Walter. Un merci tout particulier à Marc Bellein médecin généraliste qui a assuré et assure encore une grande partie des séances de simulations de consultation en médecine générale, toujours à l'écoute des étudiants et exerçant avec passion son métier, son engagement se devait d'être souligné. L'équipe du CESIM de Brest, lauréat du prix Passion Enseignement Supérieur 2016, dans la Catégorie «Formation tout au long de la vie » qui a rendu possible cette expérience pendant trois années.

\section{Former les futurs médecins à la relation avec les patients par la simulation}

1 La réglementation concernant la formation initiale des médecins, stipule que la formation à la communication et à la relation est le premier des 362 objectifs fixés aux études de médecine (Jorf, 2013). Elle concerne aussi les odontologues, les sages femmes, l'ensemble des paramédicaux du premier au troisième cycle, et la formation médicale continue (Couraud et Pruvot, 2014). Depuis 2012, l'ensemble des étudiants de la faculté de 
médecine, dans le cursus au CESIM santé de Brest passe par plusieurs simulations médicales. L'une d'entre elles est inscrite dans le cadre de la médecine générale. Les étudiants sont confrontés à des patients simulés, ils sont filmés puis débriefés par un binôme (un médecin, généraliste et un docteur en psychologie). C'est un exemple très concret de ce que peuvent apporter les SHS à un cursus scientifique dont la plupart des applications supposent une relation de qualité. Ce premier retour d'expérience pédagogique (qui se poursuit) est réalisé sur les 4 premières promotions de 180 étudiants. Il intéresse au premier plan les médecins qui prêtent leurs concours à la formation des étudiants mais aussi les universitaires des sciences de l'éducation qui doivent élaborer et participent à ce type de formation. La Formation Médicale Continue est tout aussi concernée par la simulation. Nos scénarios de simulations sont élaborés avec l'aide de praticiens expérimentés afin de garantir leur crédibilité et leur intérêt pédagogique. Les trois situations simulées reproduites ici renvoient à des situations courantes en médecine; non problématiques sur le plan strictement biomédical mais délicates quant aux dynamiques relationnelles et émotionnelles qui se produisent dans l'interaction avec le patient au plus près des conditions pratiques de la médecine générale. Ici les Sciences Humaines et Sociales sont déterminantes pour améliorer la professionnalisation des futurs médecins. Ce travail intéresse au premier plan les étudiants et les enseignants, mais aussi les seniors, maîtres de stage et praticiens qui dirigent la formation médicale. Les textes règlementaires institutionnalisent un changement paradigmatique dans l'enseignement et la formation (Kuhn, 1973) par l'introduction de compétences et de rôles du soignant qui devient explicite dans les référentiels du DFASM, (Diplôme de formation approfondie en sciences médicales) de l'HAS et du DPC mais aussi par le changement du statut de l'erreur qui passe dans la simulation du statut d'évènement « indésirable » avec recherche de responsabilités, au statut « d'occasion pédagogique». L'exercice pratique des rôles et compétences que doit assumer le médecin est facilité ici par la disponibilité croissante des ressources technologiques ${ }^{1}$ et la généralisation de la simulation dans le cursus médical (Boet et al., 2013). La simulation est désormais considérée comme une ressource didactique de premier plan dans la littérature (McGaghie et al., 2010 ; Okuda et al., 2009). Elle facilite le transfert des apprentissages, offre des possibilités d'expérimenter des situations à forts enjeux, vital, émotionnel, éthique (annonce de mauvaise nouvelle, toucher pelvien, arrêt cardio-respiratoire ${ }^{2}$, conflit au bloc opératoire, Balez et al. 2017 ; De Vries et al.. 2017) dans des conditions sécurisées (pour le patient comme l'apprenant). La création de près de 40 centres de simulation depuis 7 ans en France est significative ${ }^{3}$. Enfin c'est un champ de recherche, avec la création d'une société savante en France (la SoFraSims) et en Europe (la SESAM).

\section{1. Quand, comment et quoi simuler dans le cursus médical ?}

2 Comment la formation initiale peut-elle entraîner concrètement le futur praticien à la communication et à la relation dans sa future pratique quotidienne? Que doit-on transmettre à l'étudiant dans ces situations courantes où les compétences relationnelles du médecin comptent autant que ses savoirs médicaux? Il est en soi, intéressant de confronter à des situations problématiques concrètes et courantes, des praticiens expérimentés et des praticiens en apprentissage ; mais comment organiser cette confrontation et que peut-on en attendre? 
3 Quand introduire la simulation dans la formation médicale (McGaghie et al. 2010), que doit-on simuler, et comment? Sur la base de quelles méthodes et avec quelles évaluations (Kirkpatrick, 1994). Par les SHS notre objectif sera la mise en place de différents apprentissages pratiques par la simulation. Ce retour d'expérience permettra de répliquer ou d'adapter notre dispositif à d'autres lieux de formation.

\section{2. Un retour d'expérience pour les intervenants en Sciences Humaines et Sociales (SHS) en formation médicale}

Des formations à la relation soignant - soigné existent en France, souvent elles reposent sur les textes mais leurs élaborations pratiques dépendent d'initiatives locales. Nous souhaitons partager le travail mené au CESIM santé de Brest. La formation présentée ici est pleinement intégrée aux cours magistraux et aux stages, elle s'inscrit dans un ensemble de "simulations relationnelles » en complément des "simulations techniques » et concerne toutes les promotions par petits groupes de 9 à 12 . Chaque promotion effectue un programme de simulation à difficulté croissante qui sera brièvement exposé. Il est suivi par petits groupes en L3, M1 (exposée ici), puis en M2 et année 6. Chaque fois, un docteur en psychologie sociale et un médecin (plusieurs spécialités sont représentées selon la situation concernée) interviennent en binôme. D'autres simulations, à caractère strictement technique (par exemple : suture) ou mixte (par exemple : analyse d'image et annonce de mauvaise nouvelle, conflit au bloc) sont également délivrées aux étudiants. L'objectif est double : former à la médecine (acquisition et mise en œuvre de savoirs) et à « être » médecin (rôles et mise en œuvre de savoir faire). De fait, dans ces formations, les sciences humaines et sociales sont présentes, au même titre que les sciences biomédicales.

\section{3. Se former à la médecine}

5 Se former à la médecine c'est acquérir une immense quantité de connaissances, de savoirs et de compétences. L'arrêté relatif au deuxième cycle des études médicales (Jorf, 2013) présente dans son préambule la mention «La progression très rapide des connaissances impose des choix et conduit à rejeter toute idée d'exhaustivité dans le programme de ces trois années ». Le renouvellement des savoirs médicaux est souvent estimée à cinq ans (Shojania et al. 2007), soit une durée inférieure à la formation médicale de base (hors spécialités). Dans certains secteurs, la « demie vie » des savoir médicaux est inferieure à 70 jours. De plus, le texte de référence ne décline pas moins de 362 objectifs terminaux. Le premier nous intéresse en particulier, il stipule que la formation à la relation et à la communication est un objectif en soi.

6 La relation médecin-malade dans le cadre du colloque singulier ou au sein d'une équipe, le cas échéant pluri-professionnelle. La communication avec le patient et son entourage. L'annonce d'une maladie grave ou létale ou d'un dommage associé aux soins. La formation du patient. La personnalisation de la prise en charge médicale. (Il doit donc pouvoir):

7 - Expliquer les bases de la communication avec le malade, son entourage et la communication interprofessionnelle;

8 - Établir avec le patient une relation empathique, dans le respect de sa personnalité, de ses attentes et de ses besoins; 
- Se comporter de façon appropriée lors de l'annonce d'un diagnostic de maladie grave, de l'incertitude sur l'efficacité d'un traitement, de l'échec d'un projet thérapeutique, d'un handicap, d'un décès ou d'un évènement indésirable associé aux soins;

11 - Favoriser l'évaluation des compétences du patient et envisager, en fonction des potentialités et des contraintes propres à chaque patient, les actions à proposer (à lui, ou à son entourage) : éducation thérapeutique programmée ou non, actions d'accompagnement, plan personnalisé de soins... (JORF, 2013. p.2). Contrairement aux techniques et aux artefacts qui évoluent sans cesse, ces objectifs sont poursuivis en eux-mêmes par le soignant tout au long de sa carrière. La "relation médecinmalade " avec une équipe, "de communication avec le patient et son entourage » avec un accent sur "l'annonce d'une maladie grave ou létale ou d'un dommage associé aux soins (...) d'échec thérapeutique" (...) de communication avec le malade (...) interprofessionnelle (...) de comportement approprié (...) de relation empathique avec le patient, tout cela en vue d'élaborer un «programme de soins personnalisé» en fonction des contraintes propres au patient. Ce premier objectif met particulièrement l'accent, non sur l'acquisition de savoirs biomédicaux, mais sur l'exercice de rôles que devra assumer le médecin. Ils impliquent les compétences relationnelles et émotionnelles, autant que les compétences techniques. L'entrainement des praticiens dans la simulation de la troisième à la cinquième année vise précisément ces acquisitions.

\section{4. Se former à être médecin}

12 «Être » médecin, c'est se confronter simultanément à des problématiques biomédicales et des enjeux psychosociaux. La relation contextuelle instaurée avec le patient et ses proches est déterminante pour la prévention comme pour la prise en charge. Savoir communiquer, c'est-à-dire écouter et répondre, quelquefois négocier avec un patient afin qu'il modifie un comportement pathogène et/ou adopte un comportement immunogène. Dans ce cadre, il est nécessaire de savoir interroger la pertinence d'une prise en charge. La communication entre le praticien et le patient est fondamentale, elle est le socle de l'alliance thérapeutique qui détermine la mise en œuvre, voire la réussite, d'un programme de soins. L'activité en question ne va pas de soi dans la pratique. Le médecin doit mobiliser et combiner de nombreux savoirs (connaissances déclaratives), savoir-faire (connaissances procédurales), faire-savoir (informer) et des savoirs être (compétences non techniques) comme l'empathie (Balez et Berthou, 2014). Tout cela dans un contexte émotionnellement chargé, avec des enjeux multiples, enjeux de vie, voire enjeux vitaux, mais aussi enjeux de santé publique et d'économie de la santé. Dans ce but, nous avons élaboré des situations médicales relationnelles complexes dans l'espace de la rencontre médecin/patient au cabinet médical.

\section{5. Choix de l'espace du travail médical.}

13 Dans la simulation, les étudiants médecins expérimentent ce contexte au plus près des conditions réelles (Frenay et Bédard, 2004) qu'ils vivront en stage et dans leur carrière ${ }^{4}$ (Ong, 1995) avec des patients et des proches, ici dans le cadre d'un cabinet de médecine générale. La simulation relationnelle, intègre des aspects complexes (entrer en relation, obtenir des informations parfois sensibles, délivrer un diagnostic...etc) et transdisciplinaires (cardiologie, oncologie, psychologie...etc). Elle nécessite pour l'étudiant la mobilisation coordonnée de plusieurs registres de connaissances: 
déclaratives, procédurales / énactives et conditionnelles (Tardif, 1992). Après la simulation d'autres dimensions de la pratique sont évoquées, et discutées, de l'arrangement spatial du mobilier (et ce qu'il implique pour la relation avec le patient) au réseau d'autres professionnels et intervenants dans les soins (familles, psychologues, infirmières, assistantes sociales, sages-femmes, kinésithérapeutes, ergothérapeutes, spécialistes etc).

Nous évoquons ici des éléments primordiaux du dispositif du commencement (prébriefing) à sa fin (débriefing). L'expertise médicale collective suscitée et recueillie dans ces situations simulées, sera l'objet de publications médicales à venir, sous la forme de " conseils» pour chacune des trois situations traitées. Ici, nous décrirons la simulation relationnelle située dans le cursus, la préparation des étudiants pour susciter l'expression de compétences relationnelles au moyen d'un cadre précis. Ensuite nous décrirons le matériel (les familles de situations) et nous restituerons les retours des apprenants.

\section{La simulation relationnelle}

15 La "simulation relationnelle ${ }^{5}$ est menée dans le cadre d'un cabinet de médecine générale dans des situations où la problématique médicale se confond avec son contexte psychologique et sociologique. Ce qui se joue ici, c'est la relation avec le patient et ses proches. La qualité relationnelle déterminer la réussite ou l'échec de la thérapeutique à engager. Nous confrontons les futurs praticiens à trois simulations relationnelles proches de situations qu'ils rencontrent tout au long de leurs carrières : un(e) patient(e) : 1) angoissé(e) et en danger 2) qui ne se sent pas malade et présente des comportements dangereux et 3) avec des signes préoccupants mais qui refuse les examens pour des raisons idéologiques.

Cet ensemble de simulation se situe dans la cohérence du cursus médical.

\section{1. Une formation inscrite dans un ensemble}

La formation exposée ici est une progression à difficulté croissante de simulation relationnelle, elle complémente et s'intègre dans les programmes techniques sur mannequins, simulateurs ou dans des environnements virtuels. L'ensemble vise des compétences intégrées en santé (Parent et Jouquan, 2015). Les sessions font intervenir des médecins seniors de diverses spécialités ${ }^{6}$ et un docteur en psychologie sociale qui coordonne le projet. Elles suivent des mises en situation qui commencent dès la licence (sur la prise de décision médicale et l'annonce) et précèdent d'autres journées du même type initiées dans les années suivantes en onco/hématologie.

17 Cette formation liée à la médecine générale est restée relativement stable dans sa mise en œuvre. L'effectif des groupes d'étudiants a toutefois diminué (de 14 pour la première et la seconde promotion à 9 en moyenne) et le cadre technique du CESIM santé s'est amélioré (acquisition d'équipements médicaux pour le cabinet et caméras HD sous différents angles). Autre changement notable, la simulation est passée de la cinquième à la quatrième année afin d'équilibrer les différents programmes dans le cursus. Depuis 2012, les étudiants suivent plusieurs simulations relationnelles. En troisième année, ils doivent prendre une décision collective difficile dans un contexte d'urgence relative et de refus de soins. En quatrième année a lieu la formation de simulation en médecine générale détaillée ici. En cinquième année, une autre formation intègre des examens cliniques et 
échographiques, de la lecture d'images et des consultations simulées d'annonce de mauvaises nouvelles (Balez et Berthou, 2013; Balez et al. 2014). Enfin, en sixième année, une simulation de prise de décision collégiale, difficile sur le plan éthique, en contexte interprofessionnel (médecins et infirmiers en fin de cursus (Balez et Cozian, 2015) avec une annonce destinée aux proches, un conflit interprofessionnel au bloc (Balez et De Vries 2017). Par ailleurs, dans des cursus voisins (Sages Femmes, Odontologues), et dans certaines spécialités médicales (Touffet et al. 2015) et en DU (« Urgence en cabinet ou au domicile par exemple») la faculté programme des simulations similaires. Enfin un séminaire centré sur la relation est également animé depuis plusieurs années dans le cadre de la formation continue par le Pr Boles et madame le Bris-Benaim (formatrice et cadre de santé).

\section{2. Préparation des étudiants}

La préparation de la simulation commence sous la forme familière (et sécurisante) d'un cours magistral : il apporte des connaissances, mais surtout présente le dispositif et pose le cadre de l'exercice au plan réglementaire, juridique (droit à d'image) professionnel, relationnel et émotionnel. Un bref rappel de ces éléments précède immédiatement la séquence qui est toujours suivie d'un débriefing, moment clé, de l'apprentissage, il favorise l'émergence d'une expertise individuelle et collective. Ici la problématique est médicale, mais la difficulté se situe dans la relation au patient et à ses proches. L'organisation préalable prévoit des objectifs, des méthodes et des limites à la simulation. Nous présentons aussi le mode d'évaluation choisi en fonction des objectifs visés.

Les scénarii sont élaborés avec les médecins pour leurs assises médicales et écologiques ${ }^{7}$, puis joués par les étudiants sous forme de courtes séquences de 15 à 20 minutes dans un cabinet médical. L'ensemble est filmé sous plusieurs angles simultanément. L'écoute, la reformulation et l'usage du cadre médical voire, son explicitation, permettent de repérer comment s'établit un contrat thérapeutique et l'engagement des démarches de soins appropriés via l'expression des compétences relationnelles du médecin.

\section{3. Susciter l'élaboration et l'expression de compétences relationnelles}

Développer des compétences relationnelles et émotionnelles, faciliter leur expression pour permettre leur transfert en situation professionnelle (Frenay et Bédard, 2004) est notre objectif principal. La méthode donne quelques aperçus des contenus et instructions apportés aux futurs médecins en vue de réaliser les simulations dans un cadre performant et bienveillant (aux plans professionnel et personnel).

\section{4. La présentation : apport de connaissances et assises théoriques du cadre}

21 Le docteur en psychologie sociale, qui assistera à toutes les simulations, délivre un cours magistral de trois heures portant sur la relation et la communication médicale. Celui-ci est élaboré avec les médecins du département de médecine générale (lecture de textes portant sur la relation au patient, approche centrée sur la personne, entretien 
motivationnel, modélisation de la consultation, représentations professionnelles, savoirs profanes sur la maladie).

Les supports renvoient aux thématiques mobilisées par les simulations : deuil, médecines complémentaires, rôle du proche ou usage de l'humour en médecine par exemple (Sala et al., 2002). Le cours magistral rappelle ainsi aux étudiants que l'observance et la satisfaction du malade sont indépendantes (Crane, 1997), le concept de triple maladie ${ }^{8}$ en psychiatrie (Kleinman, 1981), les questions ouvertes et fermées et l'approche centrée sur la personne (Rogers, 1961). En somme, nous abordons en amont les notions qui semblent souvent trop théoriques aux étudiants mais qui reviendront presque systématiquement au débriefing soulignant ainsi leur pertinence au plan pratique. On y visionne des simulations filmées dans d'autres formations pour les analyser et familiariser les étudiants au dispositif spatial (cabinet médical, salle d'attente) et technique (usage des caméras). On rappelle aussi les "règles du jeu» de ce qui sera un groupe centré (Kitzinger, 1994) dont le mode de débriefing sera adapté aux objectifs de la formation en santé (Rudolph et al., 2006). Les règles apportées en amont de la simulation serviront de cadre pédagogique et relationnel.

Le groupe deviendra un espace de communication qui observera et analysera les interactions et les représentations dans l'action et sur l'action menée dans la consultation en se référant à l'action médicale (Raemer et al. 2011). Les étudiants qui joueront les patients seront une ressource didactique en terme de feed-back pour celui qui joue le médecin. Ce sera aussi pour eux l'occasion d'expérimenter et de réfléchir sur la consultation médicale du point de vue du patient (nous reviendrons sur ce point dans la discussion). Les enregistrements mettent en perspective le souvenir et le vécu des observateurs comme des acteurs dans la simulation ou sur la simulation et ce qui a été filmé. Ils permettent aussi de se focaliser sur la communication orale ou non verbale par exemple et sur les distorsions entre ce qui est dit et ce qui est montré dans l'interaction médecin/patient (Merton et al. 1990 ; Caverni, 2000). Afin de renforcer la sécurité affective des participants (Boet et al., 2013 ; Raemer et al., 2011, Merton et al., 1990). Le cadre permet aux étudiants de s'exprimer librement et pour cela, il garantit le secret. Concrètement, l'erreur dans la simulation est présentée comme une opportunité pédagogique, elle est valorisée car elle bénéficie au groupe, en ce qu'elle facilite la prise de conscience des difficultés à interagir dans certaines situations. Les simulations «relationnelles» ne comportent pas d'aspect «technique». Établir une relation de qualité avec un patient est souligné comme un aspect central de l'exercice médical (Merton et al, 1990) et donc, l'objectif en soi, de la formation. Aussi le spectre des solutions est nécessairement large. Cette formation obligatoire n'est pas sanctionnante, et elle ne saurait l'être, même implicitement. Ici, la connaissance et la compétence médicale n'impliquent pas nécessairement la performance dans la relation au patient. Autrement dit, il importe moins de « réussir » ou de « rater » la rencontre simulée avec le patient que de verbaliser pourquoi, et en quoi, la relation instaurée peut-être efficace ou non sur le plan thérapeutique, indissociable du plan relationnel. En d'autres termes, il importe de déporter le conflit cognitif ou socio-cognitif entre apprenants, sur les critères de qualités de ce qu'est une prise en charge adaptée dans les situations présentées. Il importera ensuite de l'améliorer en situation réelle. On réaffirme donc qu'il y a plusieurs manières de réussir ou d'échouer dans une relation. Les problèmes de communication posés au médecin dans la simulation et dans la réalité trouveront des solutions qui seront discutées collégialement. 

ne sont en aucun cas obligés de passer par la simulation en tant qu'acteur, s'ils le font, c'est à visée propédeutique avec des directives données à l'avance et discutées individuellement avec les formateurs. Dès la préparation, comme dans le débriefing il protégera les individus, évitera les remarques blessantes, les jugements personnels, la sur-responsabilisation des acteurs. On s'attachera aux situations telles qu'elles se présentent contextuellement au médecin et au patient, à leurs comportements respectifs, non à leur personnalité réelle ou induite. Enfin, il conclura par l'information relative au droit à l'image et aux utilisations des vidéos (recherche et pédagogie). La possibilité sera systématiquement offerte aux étudiants de détruire les supports enregistrés après-coup, sans aucune justification à fournir, ceci conformément à la déontologie de la recherche sur les humains en psychologie (Caverni, 2000).

\section{5. Choix de la séquence de travail} des étudiants et en jeu : Etre filmé en tant que médecin devant des pairs et des experts est une épreuve délicate. L'autre enjeu est de recueillir et synthétiser les préconisations propres à chacune des trois situations abordées et qui seront restituées aux étudiants (Merton et al., 1990 ; Bion, 1961). Nous proposons un acronyme simple pour résumer les éléments essentiels de la planification en amont du travail en simulation $(\mathrm{ORC}+\mathrm{T})$ : pour Outils, Référents (épistémique et expérientiels), Conflit avec un « Tiers expert ». consultation).

2) Les Référents, ressources didactiques, ont une double légitimité (épistémique et expérientielle). Dans le dispositif, ils sont chargés des aspects et savoirs techniques, conditionnels, juridiques. Ils sont placés d'une part en autorités épistémiques (Bar-Tal et al. , 1991 ; Ellis et Kruglanski, 1992) et d'autre part en praticiens expérimentés. Au cours du débriefing ils peuvent par exemple évoquer leurs expériences singulières, voire être appelés à faire " aveu d'impuissance » dans certaines situations (comme une annonce de décès). Il est essentiel que cette personne soit reconnue comme un expert sur ces deux volets par les apprenants.

3) Dans la situation d'apprentissage qui se présente en simulation, les Référents contribuent à ramener le Conflit intra-individuel et socio-cognitif vers les critères de qualité du travail effectué, vers la création, le renforcement d'une communauté épistémique à partir du groupe.

4) Le "Tiers expert" spécialiste, n'est pas en mesure d'évaluer les compétences techniques (même implicitement) et donc de les sanctionner. Principal responsable de la formation, il est légitime de par sa pratique, sa formation ou son expérience (ergonome spécialisé en médecine, docteur en sciences de l'éducation avec une application dans les soins, Dr en psychologie ou en linguistique), il peut équiper les observateurs de grilles d'observation ou les orienter sur une cible spécifique (par exemple « le proche, le cadre spatial...etc). Il régule le groupe, permet aux Référents de rester dédiés à leur coeur 
d'expertise, il suscite l'aveu d'incompétence voire d'impuissance de leur part et facilite l'orientation du conflit sur les critères de qualité du travail. Selon ses compétences, il peut éventuellement retirer du dispositif, en amont ou en aval, un apprenant mis en difficulté par la situation provoquée (traumatisme, proximité personnelle au scénario etc...) sans jugement (même implicite) et sans laisser le groupe livré à lui-même.

En tant que psychologue par exemple il facilitera la reformulation des apprenants et des experts, s'assurera que tous et toutes s'expriment sans jugement.

À distance, ce tiers expert psychologue peut théoriser les évènements en simulation (relations groupales, métrologie de la performance du groupe ou des compétences non techniques comme l'empathie (Balez et al. 2014, Lelorain et al., 2013 ; Lamothe et al., 2014) et ses liens avec le burn-out (Zenasni et al., 2012). Il peut mener des entretiens de recherche individuels ou des focus groupes sur la simulation, voire valoriser scientifiquement le matériel (verbatim, vidéo, questionnaire).

Après chacune des simulations médicales relationnelles, nous choisissons des objectifs et en fonction de ceux-ci, nous élaborons des modes de débriefing.

\section{5. 1. Une analyse réflexive, rétrospective et prospective}

34 Amener les étudiants médecins à une analyse réflexive (Dewey, 1933, Schön, 1994) et rétrospective de leur propre activité (Pastré, 2006) est central. Au plan individuel, l'étudiant-acteur donne du sens aux actions envisagées, entreprises et interrompues, il conceptualise la situation (Samurcay et Rogalski, 1998) d'où l'usage systématique de la reformulation par le formateur. La vidéo est, (dans la grande majorité des cas) entièrement rediffusée. On observe le comportement verbal et non verbal (Rudolph et al. 2008 ; Knapp et Hall, 2002) et la perspective singulière de chaque acteur (le patient, le médecin, le proche etc.). Dans le groupe, il s'agit ensuite de contextualiser, décontextualiser et recontextualiser l'expérience réalisée (Kirkpatrick, 1994). La synthèse de ces observations in situ de l'analyse thématique des observations individuelles devrait améliorer les performances dans une situation similaire (Dieckmann et al., 2009) et surtout accroître la confiance des étudiants (Laschinger et al., 2008). Le questionnaire à portée réflexive individuel, à la suite de la simulation vise aussi ce but ("Qu'avez vous ressenti personnellement dans les simulations? Quelles sont les priorités dans ce type de situation et pourquoi ?).

\section{5. 2. Légitimer les affects}

Protéger les apprenants, valider et légitimer les affects est essentiel. La simulation visionnée en direct, sur un grand écran, dans la salle de débriefing sous plusieurs angles, sous le regard des pairs et enseignants, peut être déstabilisante pour les acteurs. Le débriefing sera un moment sérieux mais convivial et bienveillant, c'est un moment d'échange social, une réflexion commune autour du vécu de la situation (Dieckmann et al., 2009). C'est la fonction de l'approche centrée sur la personne (Peretti, 1974) présentée et illustrée dès la préparation. On évoque le vécu subjectif des acteurs impliqués dans la situation, puis les inférences des observateurs. La vidéo permet de revenir, dans un second temps, sur les faits observables. Comprendre les distorsions entre ce qui est vécu par les uns, ou les autres et ce qui s'est produit est central (le médecin, l'interne, le patient, proche, l'observateur). L'ensemble du dispositif est orienté pour mettre en perspective et partager autour de l'erreur bien comprise, Dire le doute et la réflexion 
professionnelle dans un cadre bienveillant et valorisant où chacun peut s'exprimer sans être interrompu ni jugé.

\section{5. 3. Légitimer l'erreur}

L'erreur en simulation est une ressource pédagogique, c'est l'occasion de s'interroger sur ce qu'elle implique en médecine. La littérature portant sur la déception dans l'expérimentation psychologique insiste sur l'importance du débriefing (Berscheid et al., 1967) La formation en simulation médicale s'intéresse aussi à cette question (Calhoun et al., 2015). Souvent les étudiants médecins confrontés à l'erreur, se trouvent en grande difficulté, la recherche d'un soutien social est difficile à envisager (Volpp et Grande, 2003). Le débriefing est une alternative aux expériences de réunions en équipe où l'erreur est particulièrement problématique (Gallagher et al., 2003). L'erreur, réelle ou supposée est une remise en cause émotionnelle du soignant par ses pairs, son administration, ses patients et lui-même (Wu, 1999). Dans la simulation relationnelle c'est l'occasion de s'interroger collectivement avec bienveillance sur ce qui peut ou doit être tenté dans ce type de situation. C'est aussi l'occasion de changer de paradigme, l'erreur ne doit pas être assumée par un seul acteur mais comprise par le collectif dans un contexte.

\section{5. 4. Elaborer une synthèse collective réutilisable des critères de qualité d'une prise en charge adaptée}

37 Cet objectif est apparu dès le début des formations. Il consiste à élaborer avec l'aide des étudiants, médecins seniors et participants une synthèse de chaque simulation comportant un référentiel écrit de «conseils» dans les situations types abordées. Ces recommandations s'inscrivent précisément dans la démarche d'expertise collective.

\section{Méthodes}

\section{1. Déroulement du débriefing et matériel}

Il est structuré en trois étapes (Rudolph et al., 2006 ; Zigmont et al., 2011) familières aux médecins formateurs. Avant d'aborder les aspects techniques ou procéduraux. 1) Les acteurs font part de leurs émotions, en particulier négatives à l'égard du patient, du proche, du collègue ou du médecin Le « NURS » pour « Nommer, Comprendre, Respecter et Soutenir » (Back et al., 2009) par exemple est une technique simple et souvent familière aux médecins pour organiser l'expression et la légitimation des émotions. Cependant, la désirabilité sociale entrave souvent cette expression vis à vis de leurs collègues médecins ou vers le patient. Les étudiants ont souvent intégré une bienveillance conformiste artificielle à l'égard du patient, même simulé, comme du collègue médecin. En effet la déontologie proscrit la critique. Le groupe d'appartenance est celui des étudiants (qui n'ont pas prêtés le serment d'Hippocrate) mais leur groupe de référence normatif est celui des médecins séniors (Hyman, 1968). Ne pas dire du mal des autres médecins (même en simulation) revient à montrer que l'on promeut la norme du groupe «médecins » que l'on souhaite intégrer. Cet aspect psychosocial renvoie à l'identité professionnelle visée dans le cursus de médecine, il doit être verbalisé et compris. Aussi certains affects exprimés sont légitimés. Cette norme et ses implications fonctionnelles pour les pairs médecins et les patients sont donc abordées. La simulation permet de la questionner, 
voire de la contourner : on critique éventuellement un personnage et non la personne ou le professionnel, on remet en cause une action, non une intention. Enfin, le débriefing donne la possibilité de critiquer l'ensemble du dispositif (de sa mise en place à ses implications idéologiques et pédagogiques). Les formateurs encouragent cette remise en cause, partie prenante, du processus d'apprentissage. La critique permet d'améliorer le dispositif et de progresser dans la réflexion comme nous le verrons dans les résultats observés. 2) Le groupe s'oriente vers une synthèse qui peut prendre la forme de réponses collectivement élaborées aux questions suivantes : comment définir cette situation, quels sont les enjeux, quelles sont les demandes, que peut-on tenter? Autrement dit quels seraient les critères de qualité d'une prise en charge adaptée à ce type de situation. L'expression du conflit sur l'activité du soignant au plan relationnel est donc encouragée pour élaborer des réponses collectives à ces questions. Ces interrogations sont accompagnées de reformulation d'encouragement et de temps de silence. Après cette étape, avec le concours des médecins séniors : 3) On rappelle, des notions théoriques et cliniques qui permettent de donner du sens à la simulation. Par exemple, les notions d'identification projective ${ }^{10}$ ou de la fonction apostolique du médecin ${ }^{11}$ (Balint, 1996) reviennent régulièrement. La vidéo est utilisée après les échanges avec les " acteurs ", le groupe d'observateurs et enfin les formateurs. Cette rétroaction est centrale (Issenberg et al., 2005) elle met en perspective le vécu exprimé et ce qui a été enregistré. La vidéo ${ }^{12}$ permet d'adopter des points de vue multiples (celui du patient celui du médecin) : c'est un élément essentiel reconnu pour son intérêt pédagogique en milieu clinique par exemple (Tannenbaum et Cerasoli, 2013).

Le visionnage collectif est aussi l'occasion d'entretiens qui s'apparentent à l'autoconfrontation et à la confrontation croisée (Clot, 1999). Le dialogue interroge l'action réalisée, envisagée, tentée, interrompue, la parole donnée, immédiate ou différée aux acteurs est riche (Clot et al., 2001). Ce visionnage permet d'inférer des interprétations sur la qualité et les caractéristiques de la communication et de ses finalités dans la rencontre du médecin et du patient. Les étudiants y trouvent aussi l'occasion d'échanger, avec leurs pairs, formateurs et enseignants sur leurs expériences de la formation médicale. Le débriefing est ici conçu comme une réflexion guidée, facilitée dans un ensemble d'apprentissage expérientiel (Duboscq et Clot, 2010). Précisons toutefois que ce n'est pas, pour autant, un groupe de parole. Les notes prises au cours des débriefings sont restituées à l'ensemble des étudiants en fin de formation. Ceux qui le manifestent peuvent solliciter un entretien particulier avec le psychologue.

Quand toutes ces ressources didactiques de la simulation sont épuisées nous procédons, avant d'entamer la simulation suivante, à une synthèse orale, suivie d'une courte pause. $\mathrm{Au}$ terme de la journée de formation, les étudiants rédigent leurs observations au moyen d'un questionnaire orienté sur leurs émotions et leurs réflexivités professionnelles (Bion, 1961 ; Dewey, 1933 ; Argyris et Schön, 1974).

41 Ils peuvent également donner leurs observations à propos du dispositif (anonymement ou non) Voire s'adresser au secrétariat et au doyen de la faculté.

\section{2. Acteurs et procédure}

Dans un premier temps, nous distribuons les rôles et les informations spécifiques à chaque acteur. Ici, les étudiants interpréteront un médecin senior, un interne et un patient voire un « proche » dans un cabinet de consultation. 
43 À chacun des deux ou trois "acteurs ", individuellement, on rappelle le scénario : les aspects médicaux, l'historique, les symptômes, les prises en charge possibles, les informations partagées (par certains personnages) et exclusives. Les éléments, accompagnés de supports sont expliqués à chaque étudiant séparément. Ensuite se déroule la simulation (entre 15 et 20 minutes, selon les séquences).

\section{2. 1. Comprendre et gérer la peur d'une patiente susceptible d'adopter des comportements dangereux}

Le premier scénario proposé est celui d'une femme de cinquante ans Mme Tremba, se plaignant d'une «bosse sur le sein ». Elle a fait pratiquer deux biopsies successives : la première, par un médecin sénior, revenue normale ; et la seconde, à sa demande auprès d'un interne, sans en avertir le sénior. La seconde biopsie est également normale. La patiente vient chercher ses derniers résultats. Elle est reçue par le sénior et son interne, en même temps. Mme Tremba recherche essentiellement une « certitude d'être en bonne santé » auprès du médecin. Sur le plan biomédical, « elle n'a rien », mais son angoisse la conduit à multiplier les consultations et les examens (nomadisme médical). Enfin, elle dissimule que sa sœur est décédée d'un cancer du sein, dix ans auparavant.

\section{2. 2. Comprendre et gérer la peur d'un proche et l'absence de peur d'un patient en danger}

Dans une seconde consultation, le patient présente un ACFA (Arythmie Cardiaque par Fibrillation Auriculaire), il prend irrégulièrement ses traitements, pratique des sports à risques (voile en solitaire et équitation avec sauts d'obstacles) et vient au cabinet sur l'insistance de sa compagne inquiète, après deux passage aux urgences, suite à des pertes de connaissance sans conséquences (une au cours d'une séance d'équitation, la seconde sur son lieu de travail). Le patient dissimule son intérêt professionnel et personnel à maintenir ses pratiques à risque et semble négliger totalement les évènements. Il ne craint pas la mort (qui serait la conséquence d'une chute suivie d'une hémorragie ou d'une embolie consécutive à sa non observance du traitement anticoagulant). Il pourrait cependant être inquiété par une perte d'autonomie potentielle. Pendant la consultation, il exprime une certaine défiance à l'égard du médecin («incapable de le soigner », alors qu'il «ne sent rien»), il répond au téléphone à plusieurs reprises et si le médecin ne restaure pas le cadre thérapeutique il est susceptible de quitter la consultation pour raison professionnelle.

\section{2. 3. Comprendre et gérer les réticences d'un patient à effectuer des examens}

Dans la troisième consultation, un(e) patient(e) présente des signes cliniques transitoires préoccupants elle est amené à la consultation par son/sa metteur en scène et ami. Le patient présente un essoufflement et une perte de poids importants, quelques jours avant, il a présenté une pupille gauche très rétrécie pendant quelques jours et une paupière tombante, mais ces signes ont spontanément disparus. Il faudrait faire pratiquer des examens complémentaires (prise de sang, radio pulmonaire) car le diagnostic peut être sérieux et nécessiter une prise en charge immédiate. Cependant, la situation personnelle et professionnelle du patient le conduit à refuser ces examens nécessaires. Le proche qui l'accompagne est doté d'un statut incertain (personne de confiance et employeur qu'il est nécessaire de clarifier). 


\section{Résultats} sont rapportées ci-dessous. Le questionnaire proposé après les sessions est centré sur la réflexivité professionnelle (Argyris et Schön, 1974). Deux registres sont visés dans cette approche. D'une part, les émotions contextuelles suscitées par les simulations, «Qu'avez vous ressenti personnellement dans (ou devant) cette simulation?». D'autre part, les aspects techniques généralisables à d'autres situations: Qu'avez-vous fait ou tenté, ou qu'auriez vous fait ou tenté et pourquoi? Quelles sont les priorités dans ce type de situation et pourquoi? Ces retours faciliteront la reproduction ou l'adaptation de ce travail à une autre structure de formation, ils peuvent aussi faire l'objet d'une analyse qualitative thématique, textuelle ou quantitative.

\section{1. Une expérience émotionnelle} arrive sans avoir toutes les infos (...). Le sentiment d'impuissance. F41 Je me suis sentie impuissante et frustrée, parce que malgré toute ma bonne volonté le patient évoluait très peu. F42 (...) et je me suis rendu compte que certains patients étaient très durs à gérer pour un médecin (... ).H43 De la surprise, en premier lieu. (Je me faisais « dominer » par la personnalité hystérique de ma patiente, exacerbée par les remarques de son "compagnon »). Des expériences émotionnelles déstabilisantes: F43 « On ressent de la peur, de la peine, de l'incertitude quant à l'avenir... ».

\section{2. Une expérience de décentration cognitive}

Les étudiants réalisent également l'importance thérapeutique de comprendre le point du vue du patient et ses propres représentations afin de mettre en œuvre une démarche thérapeutique. $\mathrm{F} 42$ (...) et qu'il fallait vraiment tenir compte de l'histoire de la patiente et la conception qu'elle a de la médecine, pour pouvoir augmenter ses chances (...). La patiente n'avait pas confiance en son médecin, et ne concevait pas la santé de la même manière que lui (pour la patiente, sa santé c'était son apparence physique, ses capacités d'attraction). C'est donc difficile pour un médecin de faire comprendre à tous ses patients, l'importance d'un suivi médical, car au final les patients ne se rendent pas tous compte (ou ne veulent pas le savoir, par peur) de ce qu'est une maladie et de ses conséquences; et s'inquiéteront uniquement si la maladie entraîne un gros impact sur leur activités sociales et professionnelles. Le médecin doit être capable d'entendre l'expérience du patient et de se décentrer relativement à ses propres affects $\mathrm{H} 43$ (...) Puis, il a fallu cogiter pour pouvoir démêler la situation, ce qui n'était vraiment pas évident. Puis, l'envie de réussir à convaincre la patiente de poursuivre sa prise en charge, et l'inquiétude qui allait avec ("vais-je y arriver?»). H44 Laisser le patient s'exprimer, parler de ses doutes, de ses craintes, détecter ce qui pourrait être utile afin de convaincre le patient qu'on veut l'aider (tout en respectant ses croyances et pratiques, du moment qu'elles ne le mettent pas en danger). Mettre le patient en confiance +++. Comprendre les spécificités du malade pour percevoir les approches relationnelles qui seront les plus efficaces. Le but étant de le faire coopérer et collaborer au 
maximum et pouvoir entamer une vraie relation thérapeutique. H45 Nécessité d'alliance patient/ médecin le plus vite possible (...).

\section{3. Une expérience d'adaptation cognitive et émotionnelle}

51 Ces simulations et retours d'expérience sont aussi l'occasion de réaliser que la réussite de leur future activité dépend de cette adaptation relationnelle aux problématiques des patients. F42 (...) qu'on a tous intérêt à essayer dès maintenant de trouver des astuces, et de s'interroger sur la question de COMMENT faire passer notre message à nos patients de façon à ce qu'ils comprennent notre prise en charge (...) en tant que médecin, on n'avait pas le contrôle et on n'était pas maitre de cette prise en charge, qu'il fallait avant COMPRENDRE le patient, et ADAPTER notre façon d'introduire la prise en charge au cas par cas... Mais aussi que la relation peut littéralement basculer dans le bon ou le mauvais sens et que le médecin doit intégrer son propre état émotionnel face au patient car il impacte la relation thérapeutique. H44 (...) à de nombreux moments durant une consultation la $R M M^{13}$ peut basculer d'un bout à l'autre: être très conflictuel puis devenir une alliance efficace en un clin d'oeil. La maîtrise de ses nerfs est importante pour ne pas s'énerver trop vite ou pour céder à la panique devant le patient. F43 c'était très intéressant. Quand j'ai joué le médecin je me suis vraiment retrouvée dans une impasse, je ne savais plus comment me sortir de cette situation. C'était très agaçant de ne pas réussir à se faire entendre, surtout sur un sujet aussi important. H42 Garder son calme. Ne pas paniquer. Réussir à rester concentré, face aux différentes remarques ayant pour but de me perturber. F45 Il faut que nos émotions nous guident dans la prise en charge qu'on fait des patients car on est des humains et la médecine n'a pas réponse à tout, il faut parfois laisser aller nos émotions.

\section{4. La relation en simulation est une source de satisfactions multiples pour les étudiants}

Enfin les étudiants disent qu'une relation bien établie avec le patient est en soi une source de satisfaction pour le médecin, y compris en simulation. H44 Malgré tout quand ça fonctionne bien, c'est génial. Un patient qui ressort de la consultation rassuré et souriant apporte un sentiment de devoir accompli. La simple possibilité d'échanger sans crainte d'être mal jugé est quelquefois exprimée comme une expérience nouvelle par les étudiants habitués à être évalués par les seniors et par les pairs. Certains expriment l'importance des compétences relationnelles à acquérir, par et dans, l'expérience. Il est aussi question d'identité professionnelle, ils évoquent le médecin qu'ils voudraient être. F43 Plus de confiance en moi concernant le dialogue avec les patients. J'ai compris qu'il n'y avait pas de consultation idéale, que nos actions et nos paroles aideraient quelqu'un et éloigneraient quelqu'un d'autre. Et c'est pas grave. Il faut trouver le médecin qu'on veut être. H45 être patient, laisser le temps au patient de "digérer ", prendre conscience du contre transfert, rester disponible (avoir le temps, attitude physique ouverte, être attentionné).

\section{5. L'intérêt de se fixer des objectifs thérapeutiques}

Sur le plan technique, les étudiants réalisent l'intérêt de fixer des objectifs thérapeutiques à la consultation, objectifs qui peuvent être de l'ordre de la relation. H41 La priorité est de protéger la patiente, d'éviter le nomadisme médical. F44 J'ai tenté de lui faire comprendre l'intérêt de ce rendez-vous, j'ai évalué les modifications de son mode de vie, suite à 
l'hospitalisation, je lui ai expliqué les enjeux et les risques de ne pas prendre son traitement, j'ai essayé de lui faire voir l'inquiétude de sa femme, et je lui ai proposé un rendez-vous pour qu'il se sente impliqué dans un parcours de soin.

\section{Discussion, prolongements et conclusion : la Simulation, les SHS comme ressources pratiques}

54 Tous les étudiants qui se sont exprimés disent une profonde satisfaction ; Qu'ils aient été acteurs : H4 C'était une superbe expérience, et je me suis senti très immergé dans mon personnage durant ce jeu de rôle (ce qui rend la situation plus intense); Ou observateurs : F43 Regarder était tout autant enrichissant. F45 J'ai trouvé cela très bien car on se voit en miroir, c'est comme si on se voyait parler au patient donc on voit nos erreurs et nos qualités, ce qu'il y a à améliorer. Le terme de « miroir » est souvent utilisé, le futur praticien a l'occasion de se voir à l'œuvre, ce qui l'engage dans un processus réflexif sur sa propre pratique. C'est aussi l'occasion d'expérimenter l'altérité dans la simulation. « ... nous sommes vraiment pris au jeu. Nous arrivons à ressentir ce que peuvent ressentir les patients dans les situations de consultation et d'annonce (incompréhension, non écoute, buts différents entre patient et médecin). Etre mis à la place du patient nous permet de savoir ce qu'il peut ressentir et ses attentes, qui ne sont pas forcément les mêmes que le médecin.

Nous vous avons présenté notre expérience à Brest sur la formation à la relation médecin malade pour les internes en médecine générale. D'autres initiatives de formation à la relation médecin - patient existent également en France. Ce type de formation est centré sur des problématiques concrètes au plus près des réalités quotidiennes du médecin et de ses besoins, elle prend en compte l'ensemble des facteurs qui conditionnent son travail (degré d'urgence, environnement spatial, réseaux de soins, contexte thérapeutique etc). La simulation est une réponse pédagogique qui sera privilégiée pour garantir une formation médicale initiale ou continue pertinente (Académic médecine, 1999) dans un contexte sécurisé pour les apprenants comme pour les patients. Ces dispositifs devraient améliorer les performances dans une situation similaire au moins (Dieckmann et al., 2009).

\section{Cinq points sont à retenir pour les prolongements de cette expérience pédagogique}

56 - La validité de cette méthode doit pouvoir se mesurer dans l'exercice autrement que par la satisfaction des apprenants (Kirkpatrick, 1994), ceux-ci déclarent avoir appris quelque chose de l'ordre de la réflexivité professionnelle et que cela impactera leurs futurs comportements professionnels. À notre sens, il faudrait aussi prendre en considération le point de vue du patient.

Une démonstration empirique sur le moyen et long terme reste à mener.

- Les étudiants jouant les patients et sont sollicités en tant que ressources didactiques (en terme de feed-back pour ceux qui jouent le médecin, le patient, le proche ou les observateurs). C'est une question en soi car les simulations médicales tendent plutôt à faire appel à des patients simulés, comédiens professionnels et parfois des patients « experts ». Nous pensons que dans le cadre de la formation initiale, prendre la place du 
«patient » ou du «proche » comporte un intérêt propédeutique en soi et c'est aussi ce que déclarent nos apprenants mais ceci reste à vérifier.

- La confrontation avec les référents médecins et le tiers expert est aussi l'occasion d'adopter une démarche qualitative pour élaborer des conseils permettant de capitaliser l'expertise produite par la confrontation de plusieurs professionnels et d'apprenants sur des situations types. En ce sens notre dispositif pourrait directement modifier les pratiques des professionnels.

60 - La rediffusion presque systématique de la vidéo n'est pas l'usage habituel en simulation médicale. En général la vidéo est « marquée » par les formateurs focalisés sur des aspects précis pendant la simulation. La vidéo est aussi utilisée pour lever une ambiguïté dans la perception des apprenants. Pour notre part, le visionnage des séquences nous semble très riche car il mobilise l'ensemble des apprenants sur l'intégralité de la situation. Il permet de se focaliser sur les interactions non-verbales, ou seulement verbales, par exemple ou de permettre aux «acteurs » de verbaliser un état émotionnel ou une action envisagée, mais non réalisée à un moment précis. Là encore, il n'est pas démontré que le mode de diffusion des séquences adopté améliore le débriefing ou les performances mais c'est une piste à approfondir.

61 - Nous avons opté pour un cadre d'évaluation à visée formative, non sanctionnante (comme ce peut être le cas dans les études médicale en Suisse par exemple). Ceci afin de rassurer et sécuriser les apprenants. Il est toutefois possible qu'à l'avenir, la simulation médicale puisse adopter tantôt un cadre de formation, tantôt dans un cadre d'évaluation comme c'est le cas dans l'aéronautique par exemple.

62 Ces points sont autant de prolongements possibles à cette première série d'observations et appellent à d'autres études mais il reste intéressant de signaler qu'ils renvoient autant à la simulation en formation initiale qu'à la formation continue des soignants.

\section{BIBLIOGRAPHIE}

Académic médecine, (1999). Une présentation développée des principes fondamentaux d'optimisation de l'impact d'une FMC. vol. 14 decembre.

Argyris C, Schön D (1974). Theory in practice : increasing Professional effectiveness

Back, A., Arnold, R., and Tulsky, J. (2009). Mastering communication with seriously. San-Francisco : Jossey-Bass Publishers. ill patients : Balancing honesty with empathy and hope. New York, NY : Cambridge University Press.

Balez, R., de Vries, P., Cousin, I., Martin, E., Merieult, T. (2017). Evolution permanente des outils et procédures de travail au bloc opératoire : la nécessaire formation au travail en équipe en simulation médicale comme réponse organisationnelle. Psychologie du Travail et des Organisation.

Balez, R., Berthou, C., Carpentier, F-G. (2014). Annoncer un lymphome, la question de l'empathie des étudiants médecins. Psycho-Oncologie, 8, 29-36. 
Balez, R. et Berthou, C. (2013) Un entraînement de groupe systématique à l'annonce du cancer dans le cursus des médecins ; un retour d'expérience. Psycho-Oncologie, 7, 250-257.

Balez, R. et Cozian A-L. (2015). L'interprofesionnalité en simulation paramédicale et médicale : du groupe d'experts au groupe expert (apport de la psychologie Sociale et de la psychologie du travail et des organisations au travail en équipe de soin). $4^{e}$ Colloque Francophone de Simulation en Santé, Paris, Institut Pasteur, 4-5 février 2015.

Balint M. (1996) Le médecin, son malade et la maladie. Paris : Payot.

Berscheid, E., Abrahams, D., and Aronson, V. (1967). Effectiveness of debriefing following deception experiments. Journal of Personality and Social Psychology, 6, 371-380.

Bar-Tal, D., Raviv, A., et Brosch, A. (1991). Perception of epistemic authority and attribution for its choice as a function of knowledge area and age. European Journal of Social Psychology, 21, 477-492.

Bion, W-R. 1961. Experience with groups and other papers, Londres : Tavistock publications.

Boet, S., Granry, J-C., Savoldelli, G-L. (2013). La simulation en santé : De la théorie à la pratique. Paris : Springer-Verlag; 2013.

Calhoun, A-W., Pian-Smith, M-C-M., Truog, R-D., Gaba, D-M., Meyer, E-C. (2015) Deception and Simulation Education. Simulation in Healthcare : The Journal of the Society for Simulation in Healthcare. 10, 163-169.

Caverni, J-P. (2000). Un code de conduite des chercheurs en psychologie. Bulletin de Psychologie. $445: 49-51$.

Clot, Y. (1999). La fonction psychologique du travail. Paris : Presses Universitaires de France.

Clot, Y., Faïta, D., Fernandez, G., Scheller, L. (2001). Les entretiens en autoconfrontation croisée : une méthode en clinique de l'activité. Éducation permanente, 146, 17-27.

Couraud, F., Pruvot, F-R. (2014). Propositions pour une restructuration du troisième Cycle des études Médicales.

Crane, J-A. (1997). Patient comprehension of doctor-patient communication on discharge from the emergency department. The Journal of emergency médicine, 15, 1, 1-7. D

De Vries, P., Balez, R. Cousin, I. Martin, E. Merieult, T. (2017) La simulation inter-professionnelle : l'apprentissage des compétences non techniques. Archives de Chirurgie Pédiatriques.

Dewey, J. (1933). How We Think, édition révisée. Boston : Heath.

Dieckmann, P., Molin Friss, S., Lippert A., Ostergaard, D. (2009). The art and science of debriefing in simulation : ideal and practice. Medical teacher. 31(7), 287-94

Duboscq, J., Clot, Y. (2010). «L'autoconfrontation croisée comme instrument d'action au travers du dialogue : objets, adresses et gestes renouvelés ». Revue d'anthropologie des connaissances, 4, 2, 255-286. DOI : $10.3917 / \mathrm{rac} .010 .0255$

Ellis, S., Kruglanski, A-W. (1992). Self as an epistemic authority : Effects on experiential learning. Social Cognition, 10, 357-375.

Fanning, R. Gaba, D. (2007). The role of débriefing in simulation based learning. Simulation in Healthcare, 2(2),115-25

Frenay, M., Bédard, D. (2004). Des dispositifs de formation universitaire s'inscrivant dans la perspective d'un apprentissage et d'un enseignement contextualisés pour favoriser la construction de connaissances et leur transfert. In Presseau A. et Frenay M. (Dir.) Le transfert des 
apprentissages : Comprendre pour mieux intervenir. (pp. 241-268). Québec : Les presses de l'université de Laval.

Gallagher, T-H., Waterman, A-D., Ebers, A-G., ; Fraser, V-J., Levinson, W. (2003). Patients' and Physicians' Attitudes Regarding the Disclosure of Medical Errors. Journal of American Medical Association 2 ; 289(8) :1001-1007. DOI :10.1001/jama.289.8.1001.

Hyman, H-H. (1968) "Reference Groups." In : David Sills (Ed.), International Encyclopedia of the Social Sciences, vol. 13, edited by Davis Sills. (pp. 353-359). New York : Macmillan Company and Free Press.

Issenberg, B. S., Mcgaghie, W., C., Petrusa, E. R., Lee Gordon, D., et Scalese, R. J. (2005). Features and uses of high-fidelity medical simulations that lead to effective learning : A BEME systematic review. Journal of Medical Teacher, 27(1), 10 - 28. DOI :10.1080/01421590500046924

JORF nº095 du 23 avril 2013, Texte n³0, Arrêté du 8 avril 2013 relatif au régime des études en vue du premier et du deuxième cycle des études médicales, NOR : ESRS1308333A

Kirkpatrick, D-L. (1994). Evaluating training programs : the four levels. Berrett-Koehler Publishers, San Fancisco CA.

Kitzinger, J. (1994a). The methodology of focus groups : the importance of interactions between research participants, Sociology of health and illness, 16, 1, 103-121.

Kleinman, A. (1981). Patients and Healers in the Context of Culture: An Exploration of the Borderland Between Anthropology, Medicine, and Psychiatry. University of California Press.

Knapp, M-L., Hall, J-A. (2002). Nonverbal communication in human interaction. 5th ed., Fort Worth : Harcourt Brace.

Kuhn, T. S. (1973). La structure des révolutions scientifique. Paris : Fayard.

Lamothe, M., Boujut, E., Zenasni, F., Sultan, S. (2014). To be or not to be empathic : the combined role of empathic concern and perspective taking in understanding burnout in general practice.

Family Practice, 15.15

Laschinger, S., Medves, J., Pulling, C., McGraw, R., Waytuck, B., Harrison, Gambeta, K. (2008). Effectiveness of simulation on health profession : Student's knowledge, skills, confidence and satisfaction, 2008. International Journal of Evidence Based Healthcare, 6, 278-302.

Lelorain, S., Sultan, S., Zenasni, F., Catu-Pinault, A., Rigal, L., Jaury, P., Boujut, E. (2013). Empathic concern and professional characteristics associated with clinical empathy in French general practitioners. European Journal of General Practice, 19, 23-28.

McGaghie, W-C., Issenberg, S-B., Petrusa, E.R. (2010). A critical review of simulation-based médical éducation research : 2003-2009. Medical Education. 44, 50-63.

Merton, R., Fiske, M., Kendall, P. (1990). The focused interview. A manual of problems and procedures, New York, Free press. [1956]

Okuda, Y., Bryson, E-O., DeMaria, S-Jr., Jacobson, L. Quinones J, Shen B, Levine, AI. (2009). The utility of simulation in medical education : what is the evidence? The Mount Sinai journal of médicine. 76, 330-43.

Ong, L-M. (1995). Doctor-patient communication : a review of the literature. Social Science and Medecine. 7, 903-18

Parent, F., Jouquan, J. (2015). Comment élaborer et analyser un référentiel de compétences en santé. Louvain-la-neuve : de Boeck. 
Pastré, P. (2006). Apprendre par l'action, apprendre par la simulation. Education permanente. 168 (3), 205-216

Peretti, A de. (1974). Pensée et vérité de Carl Rogers. Paris : Privat.

Raemer, D., Anderson M, Cheng A, Fanning R, Nadkarni V, Savoldelli G. (2011). Research regarding debriefing as part of the learning process. Journal of Society for Simulation in Healthcare. Aug ; 6 Suppl :S52-7. DOI : 10.1097/SIH.0b013e31822724d0.

Rogers, C-R. (2005). Le développement de la personne. Paris : Dunod. [1961]

Rudolph, J-W., Simon, R., Dufresne, R-L., Raemer, D-B. (2006). There's no such thing as "Nonjudgmental"debriefing : a theory and method for debriefing with good judgment. Journal of Society for Simulation in Healthcare, 1(1), 49-55

Rudolph, J-W., Simon, R., Raemer, D-B., Eppich, W-J. (2008). “Debriefing as Formative Assessment : Closing Performance Gaps in Medical Education." Academic Emergency Medicine 15 (11) : 1010-16. DOI :10.1111/j.1553-2712.2008.00248.x.

Sala, F., Krupat, E., Roter, D. (2002). Satisfaction and the use of humor by physicians and patients. Psychology and health, 17, 3, 269-280.

Samurcay, R., Rogalski J. (1998). Exploitation didactique des situations de simulations. Le travail humain, 61, 4, 333-359.

Schön, D. (1994) Le praticien réflexif : à la recherche du savoir caché dans l'agir professionnel. Montréal : Éditions Logiques.

Shojania, K-G., Sampson, M., Ansari, M-T., Doucette, S., Moher, D. (2007). How quickly do systematic reviews go out of date? A survival analysis. Annals of Internal Medicine. 147 :224-233.

Street, R-L., Buller, D-B. (1987). Nonverbal response patterns in physician-patient interactions : a functional analysis. Journal of Nonverbal Behavior. 11 :234-53.

Tannenbaum, S., Cerasoli, C. (2013). Do team and individual debriefs enhance performance? A Metaanalysis. Human Factors, 55, 1, 231-45.

Tardif, J. (1992). Pour un enseignement stratégique. L'apport de la psychologie cognitive. Montréal : les éditions logiques.

Touffet, L., Balez, R., Ozier, Y. (2015). Ecouter et dire en médecine : Former de futurs médecins à la relation par la simulation : retour d'expérience en médecine générale. $4^{e}$ Colloque Francophone de Simulation en Santé, Paris, Institut Pasteur, 4-5 février 2015.

Volpp, K-G., Grande, D. (2003). Residents' suggestions for reducing errors in teaching hospitals. The New England Journal of Medicine. $348: 851-5$.

Wu, A-W. (1999). Handling hospital errors : is disclosure the best defense? Annals of Internal Medicine. $131: 970-972$.

Zenasni, F., Boujut, E., Woerner, A., Sultan, S. (2012). Empathy and Burnout : Three hypotheses. British Journal of General practice. 62 (600), 346-347

Zigmont, J., Kappus, L-J., Sudikoff, S-N. (2011). The 3D model of debriefing : defusing, discovering and deepening. Semin Perinat. 35, 52-58. 


\section{NOTES}

1. Mannequins robots, matériel audio-vidéo, smartphone et autres objets connectés

2. Les médecins urgentistes et les anesthésistes/réanimateurs sont les promoteurs de la simulation « technique » et " des compétences non techniques » (telles que « crisis ressource management ») et les médecins généralistes s'intéressent au premier plan à la simulation « relationnelle ».

3. Le CESIM à Brest, iLumens à Paris-Descartes,, LabForSIMS à Paris Sud, SIM à Nantes, CEnSIM à Chambéry , GIS à Angers (le GIS), Presage à Lille, CSM à Nice,CPA Simu Santé à Amiens, LabSUN au CHU de Caen pour n'en citer que quelques-uns. Certains sont directement associés à un CHU et/ou une faculté de médecine et s'adressent en priorité à la formation initiale et continue, et proposent des DU (DPC) de formation de formateurs en simulation pour les personnels médicaux ou para-médicaux.

4. Dans un cadre hospitalier, les médecins assumeront entre 90000 et 130000 entretiens cliniques au cours de leurs carrières (Ong, 1995).

5. Nous opposons ce concept à la simulation médicale technique (ou le registre procédural est dominant) par exemple.

6. La grande majorité des sessions sont assurées par des médecins généralistes, mais pour des raisons de disponibilité il est arrivé que d'autres spécialistes soient présents. Il est intéressant de noter que leurs recommandations recoupent les préconisations de leurs confrères et consoeurs, et qu'ils apportent aussi des éléments nouveaux.

7. Les scénarii ont étés élaborés avec le précieux concours des médecins généralistes (Le Reste, Floch) des médecins du travail (Dr. Loddé, Pr. Dewitte, Dr. Durand-Morreau) ou d'autres spécialistes (Dr. Touffet, Dr. Querré, Pr. Boles, Pr. Berthou).

8. Kleinman distingue par exemple la maladie-disease qui renvoie à la maladie du médecin (problème biologique et/ou psychologique) et la maladie - illness, qui renvoie à celle du malade (l'expérience psychosociale de la maladie et sa signification).

9. En plus des médecins référents, certaines sessions sont accompagnées par différents professionnels du soin ou de l'enseignement (membre d'association de patient, sociologue de la santé, philosophe en éthique médicale, membre du conseil de l'ordre, formateur en IFSI).

10. Quand le médecin se représente inconsciemment son patient comme étant un alter ego et lui prête ses propres affects, pensées, attitudes....

11. "La mission ou fonction apostolique signifie d'abord que chaque médecin a une idée vague mais presque inébranlable du comportement que doit adopter un patient lorsqu'il est malade. Bien que cette idée soit rien moins qu'explicite et concrète, elle possède une immense puissance et, comme nous l'avons découvert, elle influence pratiquement chaque détail du travail du médecin avec ses patients. Tout se passe comme si tout médecin possédait la connaissance révélée de ce que les patients sont en droit ou non d'espérer : de ce qu'ils doivent pouvoir supporter et, en outre, comme s'il avait le devoir sacré de convertir à sa foi tous les ignorants et tous les incroyants parmi ses patients ».

12. Le dispositif permet par exemple de "marquer " une séquence spécifique afin de pouvoir revenir dessus dans le débriefing. Le formateur effectue ce marquage de lui-même ou si un membre de groupe le demande.

13. Relation Médecin/Malade 


\section{RÉSUMÉS}

Les «simulations relationnelles" sont assurées par un docteur en psychologie sociale et un médecin ; menées dans un cabinet de médecine générale spécialement recréé pour les étudiants médecins. Les séquences avec patients simulés sont regardées en direct, débriefées et ré visionnées. La formation est réalisée sur quatre promotions de 180 futurs médecins répartis en groupes ( 9 à 14 étudiants) de $4 \mathrm{e}$ ou $5 \mathrm{e}$ année. L'objectif est de produire une analyse réflexive et rétrospective sur l'activité du médecin généraliste tout en protégeant les apprenants. La formation exposée ici est réalisée pour quatre promotions de 180 futurs médecins répartis en groupes (9 à 14 étudiants) de 4ème ou 5ème année. L'objectif est, tout en protégeant les apprenants, de susciter une analyse réflexive et rétrospective de l'activité du médecin (avec la production d'une synthèse collective écrite sur les situations abordées).

In Switzerland, men predominate in higher education science courses. However, biology courses are attended primarily by women. The little success of affirmative actions targeting girls and women in science invites us to re-examine the way these subjects are taught in Geneva primary school. We will show that only biology seems to occupy a proper place in the real curriculum. Put science back in the general knowledge could be a way to attract women in science.

\section{INDEX}

Keywords : scientist/student, analogue modelling, Life and Earth Sciences, time/space Mots-clés : simulation relationnelle médicale, relation médicale, psychologie sociale, reformulation en médecine, apprentissage de la relation en médecine

\section{AUTEUR}

\section{RALPH BALEZ}

Université Rennes 2, Centre de Recherches en Psychologie, Cognition et Communication, Université de Bretagne Occidentale, Ethique, Professionnalisme et Santé 